\title{
HIV and the Millennium Development Goals
}

\author{
Andrew J Prendergast, ${ }^{1,2,3}$ Shaffiq Essajee, ${ }^{4}$ Martina Penazzato ${ }^{5}$
}

\begin{abstract}
${ }^{1}$ Centre for Paediatrics, Blizard Institute, Queen Mary University of London, London, UK

${ }^{2}$ Zvitambo Institute for Maternal and Child Health Research, Harare, Zimbabwe ${ }^{3}$ Department of International Health, Johns Hopkins Bloomberg School of Public Health, Baltimore, Maryland, USA

${ }^{4}$ Clinton Health Access Initiative, New York, New York, USA

${ }^{5} \mathrm{MRC}$ Clinical Trials Unit at UCL, London, UK
\end{abstract}

\section{Correspondence to} Dr Andrew J Prendergast, Centre for Paediatrics, Blizard Institute, Newark Street, London E1 2AT, UK; a.prendergast@qmul.ac.uk

Received 5 August 2014 Revised 2 September 2014 Accepted 4 September 2014

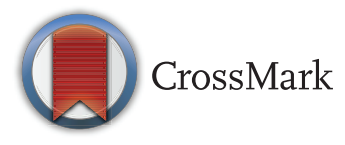

To cite: Prendergast AJ, Essajee S, Penazzato M. Arch Dis Child 2015;100 (Suppl 1):s48-\$52.

\section{ABSTRACT}

Millennium Development Goal (MDG) 6 has two HIV/ AIDS commitments: to have halted and begun to reverse the spread of HIVIAIDS by 2015 and to ensure access to treatment among all those in need by 2010. Given the almost universal lack of access to HIV testing, prevention and treatment for children in high prevalence countries in 2000, the achievements of the past 15 years have been extraordinary, fuelled by massive donor investment, strong political commitment and ambitious global targets; however, MDG 6 is some way from being attained. Prevention of mother-to-child transmission (PMTCT) services have expanded enormously, with new infections among children falling by $58 \%$ between 2002 and 2013. There has been a shift towards initiation of lifelong antiretroviral therapy (ART) for pregnant and breastfeeding women, although low HIV testing rates in pregnancy, suboptimal PMTCT coverage and poor retention in care remain barriers to achieving HIV elimination among children. Early infant diagnosis has expanded substantially but, in 2013 , only $44 \%$ of all HIV-exposed infants were tested before 2 months of age. Diagnosis of HIV, therefore, frequently occurs late, leading to delays in ART initiation. By the end of 2013, approximately 760000 children were receiving ART, leading to $40 \%$ decline in AIDS-related mortality. However, only $24 \%$ of HIV-infected children were receiving ART, compared with $36 \%$ of adults, leading to a 'treatment gap'. In this review, we summarise progress and remaining challenges in reaching MDG 6 and discuss future strategies to achieve the ambitious goals of paediatric HIV elimination and universal access to treatment.

\section{INTRODUCTION}

One of the most devastating consequences of the global HIV pandemic has been the impact on children, particularly in sub-Saharan Africa. In the absence of prevention of mother-to-child transmission (PMTCT) interventions, up to $40 \%$ of infants born to HIV-infected mothers acquire the infection, either in utero, at delivery or during breastfeeding. ${ }^{1}$ Disease progression in HIV-infected children is more rapid than in adults, with over half dying by 2 years of age without treatment. ${ }^{2}$ Even those who remain uninfected are, nonetheless, affected by HIV, with increased mortality and poor growth among HIV-exposed infants, ${ }^{3}$ and orphanhood due to parental HIV creating child-headed households in many parts of sub-Saharan Africa.

In 2000, when the United Nations Millennium Declaration was made, there were an estimated 36 million adults and 1.4 million children living with HIV; few countries had effective PMTCT interventions or antiretroviral therapy (ART) treatment programmes, and there were an estimated 500000 AIDS-related deaths per year in those aged
$<15$ years $^{4}$ (although advances in methodology led to somewhat lower estimates subsequently ${ }^{5}$ ). Millennium Development Goal (MDG) 6, therefore, focused on HIV/AIDS, among other infectious diseases, and had two principal commitments: first, to have halted and begun to reverse the spread of HIV/ AIDS by 2015; and, second, to achieve universal access to treatment for HIV/AIDS among all those in need by 2010. MDG 6 emerged from the extraordinary successes being witnessed at that time in developed countries, where the advent of combination ART was leading to reductions in mother-to-child transmission (MTCT) and HIV-related mortality, transforming HIV from a rapidly fatal infection to a long-term chronic disease. ${ }^{6}$

Enormous progress has been made in reducing the impact of HIV on children globally, although the MDG 6 targets are some way from being attained. Although 26 countries reported in 2013 that they had succeeded in reducing by half the number of new HIV infections since 2001, in other countries, urgent action is still required to halt and reverse the spread of $\mathrm{HIV}^{7}$ as $90 \%$ of new infections occur in 22 priority countries, 21 of which are in sub-Saharan Africa. ${ }^{8}$ While the number of new infections among children has fallen by $58 \%$ since its peak in 2002, mortality from HIV among children has declined by only $40 \%$ due to persistently low coverage of ART in children compared with adults. ${ }^{9}$

There is, however, a great sense of optimism that much has been achieved and that more can be done to consolidate and accelerate progress by refining the approach. The Double Dividend, ${ }^{10}$ a joint initiative between Unicef, the Elizabeth Glaser Pediatric AIDS Foundation and WHO, considers the dual goals of ending paediatric HIV/AIDS and improving child survival together, highlighting the need for an integrated approach to MDG 4 (child survival), MDG 5 (maternal health) and MDG 6. Current priorities are, therefore, focused on embedding HIV prevention and treatment within broader Maternal, Newborn and Child Health $(\mathrm{MNCH})$ services and strengthening systems for provision of more comprehensive, effective and efficient care. This approach is also endorsed in other high-level initiatives, ${ }^{8} 1011$ which together could substantially improve survival and health of women and children.

This review will summarise progress and remaining challenges within the three principal domains of the paediatric HIV prevention and treatment continuum: PMTCT to prevent HIV infection among children; early infant diagnosis (EID) and provider-initiated testing and counselling (PITC) to identify new infections; and linkage to HIV care and treatment to ensure long-term health and survival of infected children (figure 1). 
Figure 1 The paediatric HIV cascade. A continuum of care is required to connect prevention of mother-to-child transmission (PMTCT) of HIV infection with early infant diagnosis and to ensure linkage of infected children into HIV care and treatment.

\section{PMTCT OF HIV}

The generalised nature of the HIV epidemic in sub-Saharan Africa has resulted in high HIV prevalence among reproductive-aged women: by 2000, one-quarter of women presenting for antenatal care in parts of sub-Saharan Africa were HIV-infected. ${ }^{4}$ Between 2000 and 2010, HIV prevalence continued to rise in several countries, to reach a plateau of over $30 \%$ among antenatal attendees in countries such as South Africa. ${ }^{12}$ While in Europe/USA, universal HIV testing in pregnancy combined with a package of PMTCT interventions (antenatal and postnatal ART, prelabour Caesarean section and avoidance of breastfeeding) can reduce transmission to $<1 \%,{ }^{13}$ this approach is not feasible in sub-Saharan Africa, where Caesarean section is frequently unavailable or unsafe, and breastfeeding is essential for infant survival.

A public health approach to HIV prevention and treatment in sub-Saharan Africa has, therefore, been endorsed by WHO since 2001. Following the HIVNET 012 trial, ${ }^{14}$ which reported that a single dose of nevirapine to the mother and infant could reduce HIV transmission by almost $50 \%$, this became the most widely used intervention across sub-Saharan Africa, because of its feasibility and low cost. As subsequent trials reported reduced MTCT and drug resistance with combination ART regimens, the imperative to scale up more effective PMTCT interventions emerged ${ }^{15}{ }^{16}$; however, the combination of high HIV prevalence, high fertility, late or incomplete antenatal care, inadequate HIV testing rates and widespread adoption of less effective PMTCT regimens meant that by the halfway point of MDG 6 , an estimated 370000 children per year were still becoming infected with HIV. $^{17}$

From 2010, WHO recommended ART initiation from 14 gestational weeks, with lifelong treatment for women with CD4 counts $<350$ cells $/ \mu \mathrm{L}$ and two antiretroviral options for those with CD4 $>350$ cells $/ \mu \mathrm{L}$ to cover the entire period of transmission risk during pregnancy and breastfeeding: zidovudine monotherapy (Option A) or combination ART (Option B). ${ }^{18}$ In 2011, a high-profile commitment ${ }^{19}$ was made to eliminate new HIV infections among children by 2015 (defined as 90\% reduction in the number of infected children from 2009 figures, ie, to around 40000 per year) and to reduce HIV-related maternal mortality by $50 \%$. In 2013, WHO guidelines were updated to include an option for lifelong ART in pregnant and breastfeeding women regardless of clinical stage or CD4 count (Option $\mathrm{B}+)$, with the aim of simplifying PMTCT implementation; harmonising drug regimens used for pregnant and non-pregnant populations; avoiding treatment interruption, which risks selecting drug resistance and covering future pregnancies. ${ }^{20}$

By 2012, there were an estimated 1.5 million HIV-infected pregnant women globally, and PMTCT services were reaching $65 \%$ of women in the 21 priority countries in sub-Saharan Africa. $^{21}$ This expansion of effective PMTCT coverage led to new infections among children falling to 240000 in 2013, a decline of $52 \%$ from 2001 (figure 2). ${ }^{22}$ Globally, almost $80 \%$ of countries had adopted either Option B or Option B+ regimens by $2013^{22}$; however, by current projections, the ambitious target of HIV elimination is unlikely to be met, because of several remaining barriers. In 2013, for example, 54\% of pregnant women in low-income and middle-income countries did not receive an HIV test. ${ }^{22}$ Among the 22 priority countries, several still have PMTCT coverage rates below 50\% (figure 3). Further efforts are, therefore, needed to ensure that HIV testing occurs during pregnancy, to increase the pace of implementation of Option B or B+ PMTCT regimens within antenatal care services and to retain women in care throughout pregnancy and breastfeeding. As use of lifelong ART regimens for pregnant and breastfeeding women increases, the impact of PMTCT expansion on broader health services delivery will require careful evaluation. ${ }^{23}$

\section{DIAGNOSIS OF HIV INFECTION IN CHILDREN}

Diagnosis of HIV is a prerequisite for ART initiation, but serological testing in children under 18 months of age is complicated by the presence of maternal anti-HIV antibodies. Diagnosis in young children, therefore, requires nucleic acid testing, which is approximately 10 -fold more expensive, technically demanding and inaccessible in many settings. EID, together with initiation of cotrimoxazole prophylaxis for all
Figure 2 Trends in new HIV infections among children (aged 0-14) and coverage of maternal ART for PMTCT in all low-income and middleincome countries, 2001-2013. (Source: UNAIDS 2014 HIV and AIDS estimates, August 2014).

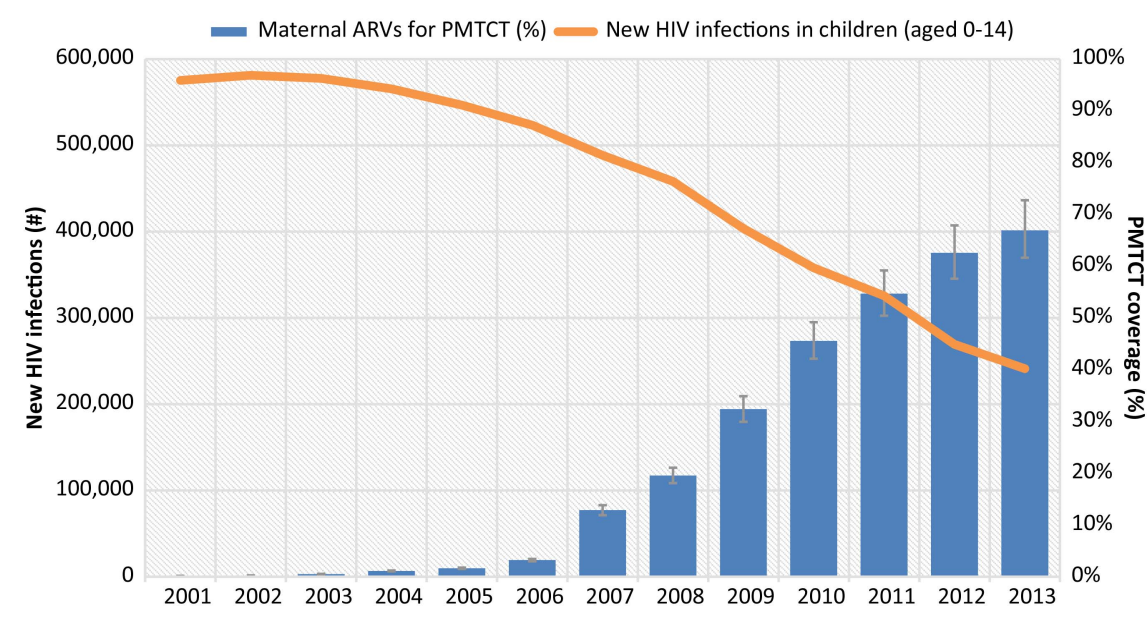


Figure 3 Global Prevention of mother-to-child transmission (PMTCT) coverage in 2013. Percentage of HIV-infected pregnant women within the 22 Global Plan priority countries ${ }^{8}$ receiving most effective regimens of antiretroviral medicines for PMTCT in 2013. (Source: Joint United Nations Programme on HIVIAIDS, The Gap Report, Geneva, July 2014 ${ }^{9}$ )

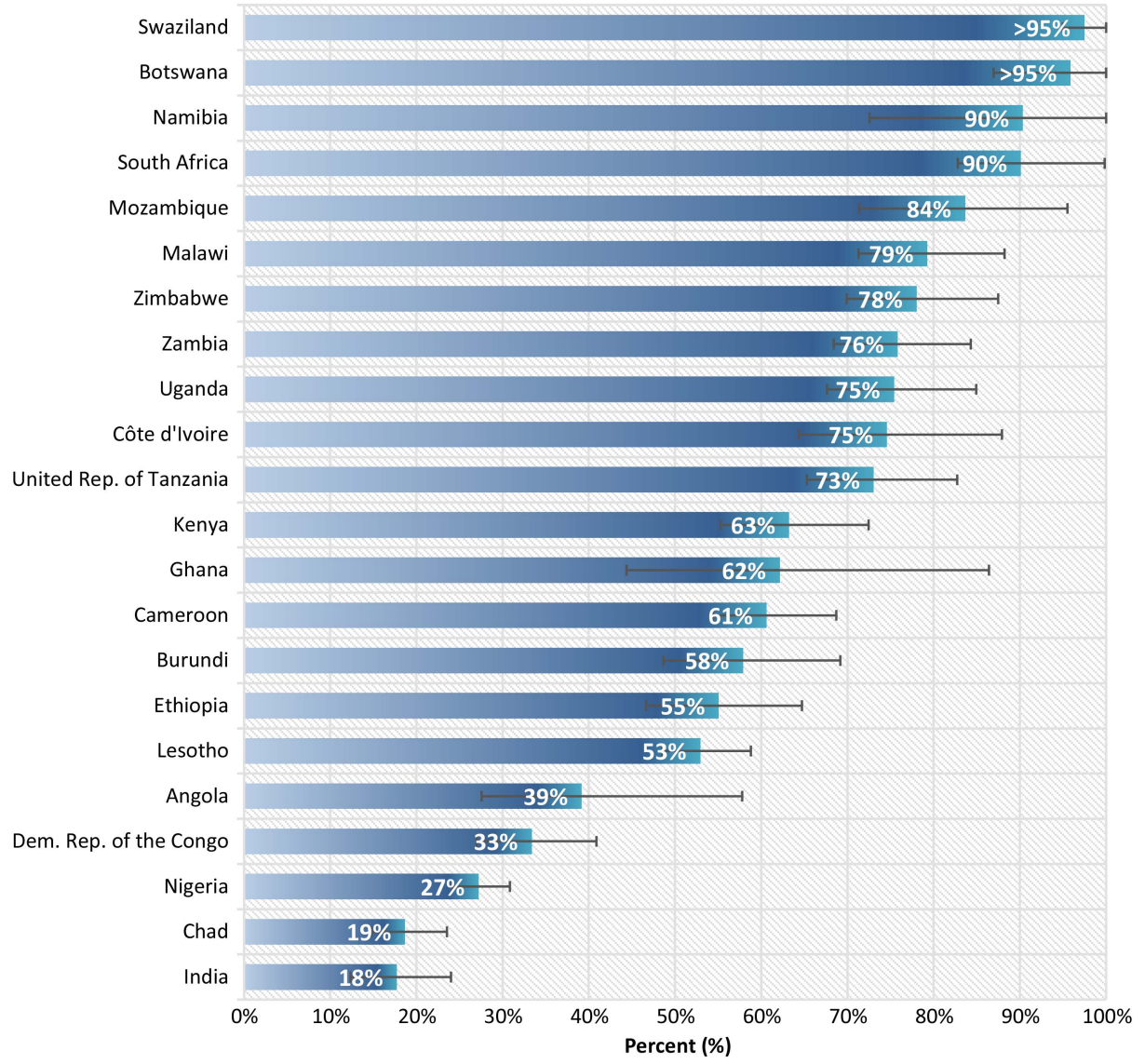

HIV-exposed infants, is recommended at 4-6 weeks of age, or at the earliest opportunity thereafter ${ }^{20}$ (although there is an emerging argument for moving the first virological test closer to birth $^{24}$ ). EID coverage has expanded substantially over the last 5 years, but in 2013, only 44\% of all HIV-exposed infants received a test before 2 months of age. Among the 22 priority countries, only 6 (South Africa, Swaziland, Botswana, Namibia, Zambia and Zimbabwe) reported coverage above 50\%, and in 5 countries (Angola, Chad, Nigeria, Malawi and Democratic Republic of Congo) coverage was still below $6 \% .^{26}$

Decentralisation of EID to rural health clinics is essential to enable integration of HIV diagnosis into a wider range of child health services, and this has been facilitated by use of dried blood spots, which simplifies collection, storage and transportation of filter paper specimens to central laboratories; however, long turnaround times and loss to follow-up remain major problems in many programmes. $^{27} 28$ Point-of-care virological testing may help to overcome these problems and improve retention in care, particularly if combined with strong referral systems, but although several platforms are in development, none has yet been rolled out. ${ }^{29}$ Diagnosis of HIV, therefore, frequently occurs late, leading to delays in ART initiation and unacceptably high infant mortality. ${ }^{30}$ An analysis of data from national programmes in Namibia, Uganda, Cambodia and Senegal showed that, despite rapid expansion of EID services, the overall effect on health remained small because in three of the four countries, fewer than $40 \%$ of HIV-infected infants actually started treatment. ${ }^{27}$

Although EID is an essential component of the PMTCT cascade, it has been argued that, in the context of limited healthcare budgets, other entry points to HIV testing are more cost-effective. ${ }^{31}$ Outside PMTCT settings, PITC in high HIV prevalence sites, such as malnutrition units, inpatient wards or within routine $\mathrm{MNCH}$ services, enables diagnosis of HIV-infected children born to previously undiagnosed women; however, opportunities for PITC are frequently missed. ${ }^{32}$ Infant diagnosis needs to be tailored to the context of an individual country, depending on available health budgets and HIV prevalence. ${ }^{31}$ To be effective, however, EID must be linked to HIV treatment and care so that children can initiate ART promptly following diagnosis. ${ }^{33}$

\section{PAEDIATRIC TREATMENT AND CARE}

HIV treatment has been one of the unprecedented success stories of recent years. A repertoire of over 25 antiretroviral drugs is now available for adults, although establishing a similar range for children has been slower. When MDG 6 was launched, there was only sporadic access to ART in sub-Saharan Africa, and the notion of universal access by 2010 seemed a highly aspirational target. Scale-up of ART provision was driven initially by the UNAIDS goal of 3 million people accessing treatment by 2005 (' 3 by 5'), and the subsequent goal of ' 5 by 10', and ART roll-out was accelerated considerably by establishment of the Global Fund To Fight AIDS, Tuberculosis and Malaria, and the US President's Emergency Plan For AIDS Relief. Although universal access to treatment remains some way off, there has, nevertheless, been an enormous expansion of ART provision: by the end of 2013, 12.9 million people globally were receiving ART. ${ }^{9}$

The first WHO guidelines for HIV-infected children were issued in $2006,{ }^{34}$ although treatment of children was included in 2003 adult guidelines. ${ }^{35}$ For many years, there was a paucity of evidence to guide management of the youngest children, but now randomised clinical trial data are available to inform 
treatment guidelines. ${ }^{36}$ In particular, the children with HIV early antiretroviral (CHER) trial in South Africa ${ }^{37}$ demonstrated the mortality reduction associated with early treatment in infancy, which led to global adoption of guidelines in 2008 recommending universal ART for HIV-infected infants. Since then, there has been a gradual shift towards earlier treatment initiation in older age groups, with 2013 guidelines advocating universal treatment of children under 5 years of age. ${ }^{20}$

By the end of 2013, an estimated 760000 children were receiving ART, leading to a $40 \%$ decline in AIDS-related mortality from its peak in 2005. ${ }^{22}$ However, only $24 \%$ of HIV-infected children were receiving ART, compared with 36\% of adults, leading to a treatment 'gap' between adult and paediatric coverage (figure 4). ${ }^{9}$ Treatment of young children is more challenging compared with adults because of high viral loads, rapid disease progression, need for appropriate formulations and reliance on caregivers, who may themselves be ill. ${ }^{38}$ Initially, liquid ART formulations were developed, but these tended to have a short shelf life and were often expensive and unpalatable. As fixed-dose combination (FDC) tablets began to be used in adults, clinicians took the opportunity to evaluate halved FDC tablets for children as an alternative to liquids, leading eventually to the development of paediatric FDCs, granules and sprinkles, which are now considered the most appropriate formulations for children.

The current focus is on treatment simplification, with increasing harmonisation across paediatric and adult guidelines; decentralisation of HIV care and treatment, with task-shifting to lower-level health facilities; and greater integration within broader $\mathrm{MNCH}$ services to enable the ultimate goal of universal treatment access to be achieved. ${ }^{20}$

\section{REMAINING BARRIERS TO MDG 6}

Given the almost universal lack of access to HIV testing, prevention and treatment for children in high prevalence countries in 2000, the achievements of the past 15 years have been extraordinary, fuelled by massive donor investment, strong political commitment and ambitious global targets. Despite these successes, there remain substantial barriers to achieving the goals of eliminating new HIV infections among children and ensuring universal access to treatment. Policymakers and clinicians have tended to ignore women and children outside PMTCT settings. Even within the PMTCT cascade, there are challenges: repeat testing, counselling and access to contraception for HIV-negative women are frequently unavailable; among HIV-infected women, retention in care and adherence to ART during the postnatal period are often poor and final ascertainment of infant HIV status frequently does not occur. Scale-up of paediatric ART still lags behind that of adults ${ }^{9}$ for multiple reasons, including low EID coverage, weak linkages to care, lack of trained staff and poor decentralisation of services ${ }^{10}$; despite availability of effective ART, therefore, three-quarters of children are not enrolled into HIV care and treatment. Among children who do initiate ART, there are limited data from many settings on ART adherence, retention within programmes and long-term outcomes. Despite simplification over time, guidelines for children remain more complex than those for adults because of differences in initiation criteria, treatment regimens and dosing across ages, leading to a perception among clinicians that treatment of children is challenging, time consuming and of low priority. Although there has been huge progress in the availability of appropriate ART formulations for children, there is need to develop more FDCs and granules, particularly for protease inhibitor-based regimens, which are now recommended first-line in young children. Finally, as HIV-infected children survive on treatment, greater attention needs to be paid to providing family centred care, addressing chronic complications of HIV infection and treatment, ensuring long-term immunological health and providing better adherence support, particularly for adolescents.

\section{THE POST-2015 LANDSCAPE}

There will continue to be a shift away from 'verticalisation' of HIV prevention and treatment programmes towards a fully integrated service delivery model, in line with the Double Dividend ${ }^{10}$ goal of supporting wider child survival efforts. This will require task-shifting of HIV diagnosis and ART provision to lower-level health facilities which, to date, has been slow to occur for paediatric care, further training of health workers, and mobilisation of communities to create a demand for services. Development of affordable and feasible point-of-care technologies (for DNA PCR, viral load and CD4 counts) for rural health clinics would ensure more timely HIV diagnosis and treatment initiation, allow monitoring of ART efficacy and reduce loss to follow-up. There will be an ongoing incentive to evaluate new antiretroviral drugs in children, particularly those from newer classes (such as the integrase inhibitors) that have no cross-resistance to existing agents and would provide the opportunity to harmonise drug choices across ages. Alternative approaches to management of paediatric HIV, such as ART initiation closer to birth, ${ }^{39}$ intensification followed by maintenance ART, early treatment initiation followed by interruption ${ }^{40}$ or short-cycle therapy (enabling time off ART during weekends), may provide clinical benefits and still be feasible within a public health approach.

New HIV infections among children continue to decline, and gradual expansion of treatment coverage is allowing more children to survive on long-term ART. The UNAIDS aspiration is for zero new HIV infections, zero discrimination and zero AIDS-related deaths. ${ }^{21}$ To this end, the UNAIDS post-2015
Figure 4 The Treatment Gap between adults and children. Percentage of adults (aged 15+) and children (aged 0-14) living with HIV receiving antiretroviral therapy in all low-income and middle-income countries, 2001-2013. UNAIDS recommends using the denominator of all adults and children living with HIV and not just those eligible for ART based on 2013 WHO eligibility treatment criteria. (Source: UNAIDS 2014 HIV and AIDS estimates, August 2014).

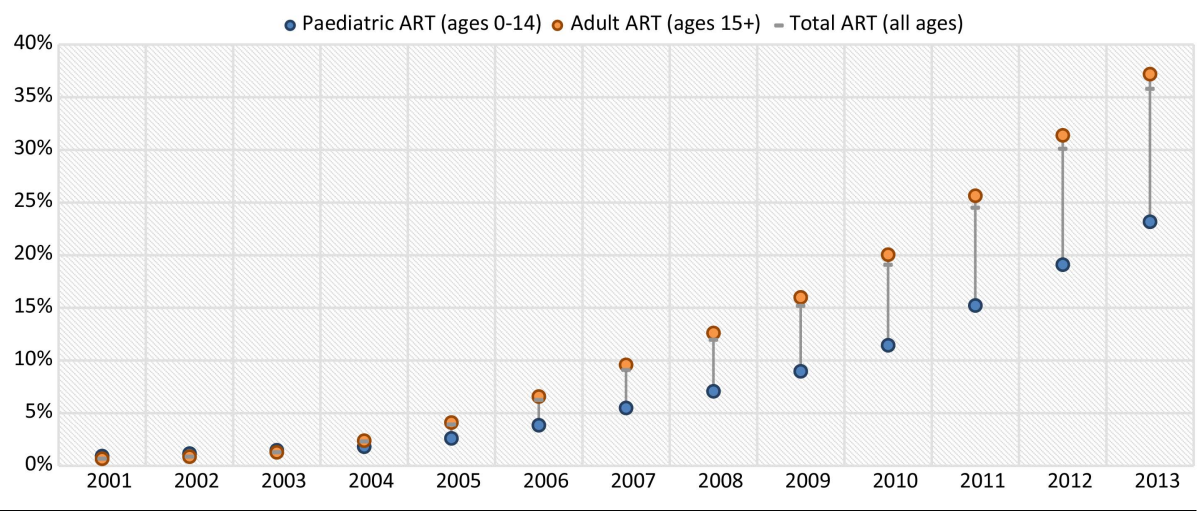


target has been labelled ' $90-90-90$ ': that 90\% of HIV-infected people will know their status, $90 \%$ of those diagnosed will be on ART and $90 \%$ of those treated will have an undetectable viral load. ${ }^{41}$ Based on modelling, UNAIDS has estimated that achieving these benchmarks by 2020 could end the AIDS epidemic by $2030 .{ }^{41}$ Although HIV/AIDS currently remains an unfinished $\mathrm{MDG},{ }^{21}$ the progress achieved in the past 15 years should galvanise the global community to capitalise on these successes and strive towards the goal of elimination.

Acknowledgements We thank Tyler A Porth, HIV/AIDS Statistics and Monitoring Officer, UNICEF, New York, for his help with data for the figures.

Contributors The first draft of this review was written by AJP following discussion with SE and MP. The draft was reviewed and edited by SE and MP in conjunction with AJP.

Competing interests None.

Provenance and peer review Commissioned; internally peer reviewed.

\section{REFERENCES}

1 De Cock KM, Fowler MG, Mercier E, et al. Prevention of mother-to-child HIV transmission in resource-poor countries: translating research into policy and practice. JAMA 2000:283:1175-82.

2 Newell ML, Coovadia H, Cortina-Borja M, et al. Mortality of infected and uninfected infants born to HIV-infected mothers in Africa: a pooled analysis. Lancet 2004;364:1236-43.

3 Marinda E, Humphrey JH, lliff PJ, et al. Child mortality according to maternal and infant HIV status in Zimbabwe. Pediatr Infect Dis J 2007;26:519-26.

4 UNAIDS. Report on the Global HIVIAIDS Epidemic. UNAIDS, June 2000. http://data. unaids.org/pub/Report/2000/2000 gr_en.pdf (accessed 29 Jul 2014).

5 UNAIDS. 2007 AIDS Epidemic Update. UNAIDS, December 2007. data.unaids.org/ pub/epislides/2007/2007 epiupdate_en.pdf (accessed 29 Jul 2014).

6 Gibb DM, Duong T, Tookey PA, et al. Decline in mortality, AIDS, and hospital admissions in perinatally HIV-1 infected children in the United Kingdom and Ireland. BMJ 2003;327:1019.

7 UNAIDS. Global AIDS response progress reporting 2014: construction of core indicators for monitoring the 2011 UN political declaration on HIVIAIDS. UNAIDS, January 2014. http://www.unaids.org/en/dataanalysis/knowyourresponse/ globalaidsprogressreporting/ (accessed 31 Jul 2014).

8 UNAIDS. Global plan towards the elimination of new HIV infections among children by 2015 and keeping their mothers alive. UNAIDS, 2011. http://www.unaids.org/en/ resources/documents/2011/name,60876,en.asp (accessed 29 Jul 2014).

9 UNAIDS. The Gap Report. UNAIDS, 2014. http://www.unaids.org/en/resources/ documents/2014/name,97466,en.asp (accessed 29 Jul 2014).

10 UNICEF. The Double Dividend: Action to improve survival of HIV-'exposed' children in the era of eMTCT and renewed child survival campaigns. December 2013. http:/ www.unicef.org/aids/files/Action_Framework_Final.pdf (accessed 29 Jul 2014).

11 African Union. Declaration of the Special Summit of African Union on HIVIAIDS, Tuberculosis and Malaria: Abuja Actions Toward the Elimination of HIV and AIDS, Tuberculosis and Malaria in Africa by 2030. Abuja, Nigeria, 16 July 2013. http://sa. au.int/en/sites/default/files/2013AbujaDeclaration.pdf (accessed 29 Jul 2014).

12 Barron P, Pillay $Y$, Doherty $T$, et al. Eliminating mother-to-child HIV transmission in South Africa. Bull World Health Organ 2013;91:70-4.

13 European Collaborative Study. Mother-to-child transmission of HIV infection in the era of highly active antiretroviral therapy. Clin Infect Dis 2005;40:458-65.

14 Guay LA, Musoke P, Fleming T, et al. Intrapartum and neonatal single-dose nevirapine compared with zidovudine for prevention of mother-to-child transmission of HIV-1 in Kampala, Uganda: HIVNET 012 randomised trial. Lancet 1999:354:795-802

15 World Health Organization. Antiretroviral drugs for treating pregnant women and preventing HIV infection in infants. WHO, July 2004. http://www.who.int/hiv/pub/ mtct/guidelinesarv/en/ (accessed 29 Jul 2014).

16 World Health Organization. Antiretroviral drugs for treating pregnant women and preventing HIV infection in infants. WHO, 2006 revsion. http://www.who.int/hiv/ pub/mtct/antiretroviral/en/ (accessed 29 Jul 2014).

17 UNAIDS. Report on the Global AIDS Epidemic. UNAIDS, August 2008. http://www. unaidsrstesa.org/resources/reports/2008-report-global-aids-epidemic (accessed 29 Jul 2014).
18 World Health Organization. Antiretroviral drugs for treating pregnant women and preventing HIV infection in infants: Recommendations for a public health approach (2010 version). http://www.who.int/hiv/pub/mtct/antiretroviral2010/en/ (accessed 31 Jul 2014).

19 UNAIDS. Global plan towards the elimination of new HIV infections among children by 2015 and keeping their mothers alive. UNAIDS, 2011. http://www.unaids.org/en/ resources/documents/2011/name,60876,en.asp (accessed 20 Jul 2014)

20 World Health Organization. Consolidated guidelines on the use of antiretroviral drugs for treating and preventing HIV infection: recommendations for a public health approach. WHO, June 2013. http://www.who.int/hiv/pub/guidelines/arv2013/ download/en/ (accessed 31 Jul 2014).

21 UNAIDS. Report on the Global AIDS Epidemic. UNAIDS, November 2013. http:/l www.unaids.org/en/resources/campaigns/globalreport2013/globalreport/ (accessed 31 Jul 2013)

22 World Health Organization. Global update on the health sector response to HIV 2014: executive summary. WHO, July 2014. http://www.who.int/hiv/pub/ progressreports/update2014-executive-summary/en/ (accessed 31 Jul 2014).

$23 \mathrm{Chi} \mathrm{BH}$, Thirumurthy $\mathrm{H}$, Stringer JS. Maximizing benefits of new strategies to prevent mother-to-child HIV transmission without harming existing services. JAMA 2014;312:341-2

24 Lilian RR, Kalk E, Bhowan K, et al. Early diagnosis of in utero and intrapartum HIV infection in infants prior to 6 weeks of age. J Clin Microbiol 2012;50:2373-7.

25 Ghadrshenas A, Ben Amor Y, Chang J, et al. Improved access to early infant diagnosis is a critical part of a child-centric prevention of mother-to-child transmission agenda. AIDS 2013;27(Suppl 2):S197-205.

26 WHO, UNICEF, UNAIDS. Global update on HIV treatment 2013: results, impact and opportunities. WHO, June 2013. http://www.who.int/hiv/pub/progressreports/ update2013/en/ (accessed $31 \mathrm{Jul}$ 2014).

27 Chatterjee A, Tripathi S, Gass R, et al. Implementing services for Early Infant Diagnosis (EID) of HIV: a comparative descriptive analysis of national programs in four countries. BMC Public Health 2011;11:553.

28 Ciaranello AL, Park JE, Ramirez-Avila L, et al. Early infant HIV-1 diagnosis programs in resource-limited settings: opportunities for improved outcomes and more cost-effective interventions. BMC Med 2011;9:59.

29 Jani IV, Meggi B, Mabunda N, et al. Accurate early infant HIV diagnosis in primary health clinics using a point-of-care nucleic acid test. J Acquir Immune Defic Syndr 2014;67:e1-4.

30 Hsiao NY, Stinson K, Myer L. Linkage of HIV-infected infants from diagnosis to antiretroviral therapy services across the Western Cape, South Africa. PLOS ONE 2013;8:e55308.

31 Penazzato M, Revill P, Prendergast AJ, et al. Early infant diagnosis of HIV infection in low-income and middle-income countries: does one size fit all? Lancet Infect Dis 2014;14:650-5

32 Kranzer K, Meghji J, Bandason T, et al. Barriers to provider-initiated testing and counselling for children in a high HIV prevalence setting: a mixed methods study. PLoS Med 2014;11:e1001649.

33 Chamla D, Mbori-Ngacha D, Newman M, et al. Evidence from the field: missed opportunities for identifying and linking HIV-infected children for early initiation of ART. AIDS 2013;27(Suppl 2):S139-46.

34 World Health Organization. Antiretroviral therapy of HIV infection in infants and children: Recommendations for a public health approach (2006 revision). WHO, 2006. http://www.who.int/hiv/pub/paediatric/infants/en/ (accessed 31 Jul 2014).

35 World Health Organization. Scaling up antiretroviral therapy in resource-limited settings: Treatment guidelines for a public health approach. 2003 revision. http://www.who.int/ 3by5/publications/documents/arv_guidelines/en/ (accessed 31 Jul 2014).

36 Penazzato $M$, Prendergast AJ, Muhe LM, et al. Optimisation of antiretroviral therapy in HIV-infected children under 3 years of age. Cochrane Database Syst Rev 2014;5: CD004772.

37 Violari A, Cotton MF, Gibb DM, et al. Early antiretroviral therapy and mortality among HIV-infected infants. N Eng/ J Med 2008;359:2233-44.

38 Prendergast AJ, Penazzato $M$, Cotton $M$, et al. Treatment of young children with HIV infection: using evidence to inform policymakers. PLoS Med 2012;9:e1001273.

39 Persaud D, Gay H, Ziemniak C, Jr, et al. Absence of detectable HIV-1 viremia after treatment cessation in an infant. N Engl J Med 2013:369:1828-35.

40 Cotton MF, Violari A, Otwombe K, et al. Early time-limited antiretroviral therapy versus deferred therapy in South African infants infected with HIV: results from the children with HIV early antiretroviral (CHER) randomised trial. Lancet 2013:382:1555-63.

41 UNAIDS. Ambitious treatment targets: writing the final chapter of the AIDS epidemic. UNAIDS, 2014. http://www.unaids.org/en/resources/documents/2014/ name,98319,en.asp (accessed 31 Jul 2014). 